\title{
3D Kinematics Analysis of Overhead Backhand and Forehand Smash Techniques in Badminton
}

\author{
Agus Rusdiana ${ }^{*}$ * \\ Sports Science Study Program, Faculty of Sports and Health Education, Universitas Pendidikan Indonesia, \\ West Java, Indonesia.
}

Submitted 04 April 2021; Accepted in final form 28 June 2021.

\begin{abstract}
Background. This study aims to analyze the movement of backhand and forehand smash stroke techniques in badminton in three dimensions using a kinematics approach. Objectives. The obtained results were analyzed using a descriptive and quantitative approach. Methods. Furthermore, 24 male badminton players from the university student activity unit with an average age of $19.4 \pm 1.6$ years, height of $1.73 \pm 0.12 \mathrm{~m}$, and weight of $62.8 \pm 3.7 \mathrm{~kg}$ participated in this study. The study was conducted using 3 Panasonic Handycams, a calibration set, 3D Frame DIAZ IV motion analysis software, and a speed radar gun. Results. The data normalization from the kinematics values of the shoulder, elbow, and wrist joint motion was calculated using the inverse dynamics method. In addition, a one-way ANOVA test was used to identify differences in the kinematics of motion between two different groups. The obtained results showed that the speed of the shuttlecock during the forehand smash was greater than that during the backhand smash. In the maximal shoulder external rotation phase, two variables were identified to have the best results during the forehand smash, i.e., the velocity of shoulder external rotation and wrist palmar flexion. Conclusion. The velocity of shoulder internal rotation, elbow extension, and forearm supination in the maximum angular velocity phase was higher when making a forehand smash.
\end{abstract}

\section{KEYWORDS: Badminton, Overhead Smash, Biomechanics, Kinematics, Three Dimensions.}

\section{INTRODUCTION}

According to Kuntze (1), stroke techniques are categorized into three types depending on the position of the racket. They include underarm, sidearm, and overhead strokes. The most frequently used attack technique is the overhead smash stroke technique (2). Similarly, there are two types of smash technique skills, i.e., forehand and backhand smash. These are powerful attack techniques, which are used to dominate the opponents and get as many points as possible; these techniques are used $39.8 \%$ of the time (3). Furthermore, smash is a fast stroke, which relies on the strength, velocity, and flexion of the wrist with the shuttlecock swooping down towards the opponent's field area (4).

The average number of smashes executed in one match in the men's single category was 69 strokes, while for the women's singles category it was 51 strokes in All England Championship 2015 (5). The world record for smash speed is held by Fu Haifeng. This medalist paired with Cai Yun, which achieved the shuttlecock speed of 332 $\mathrm{km} / \mathrm{h}$ at the June 2005 Sudirman Cup championship (6). Fu Haifeng and Cai Yun are Chinese professional men's doubles badminton players. They were men's doubles world

*. Corresponding Author:

Agus Rusdiana, Ph.D

E-mail: agus.rusdiana@upi.edu 
champions in 2006, 2009, 2010, and 2011. The shuttlecock speed exceeds that of other racket sports and reaches $493 \mathrm{~km} / \mathrm{h}$. This speed was achieved by a Chinese player Tan Boon Heong while testing a new racket product (Yonex ArcSaber Z-Slash) in 2017 (7). Meanwhile, the fastest backhand smash was achieved by Taufik Hidayat, an Indonesian player who won a gold medal at the 2004 Athens Olympics; he achieved the shuttlecock speed of $206 \mathrm{~km} / \mathrm{h}$ (5).

Backhand smash is an overhead stroke which uses the rear racket head. When performing this stroke, the body is positioned with its back to the net, and the wrist joint flexion motion is prioritized and directed to swoop backward (8). This occurs because the transfer of body weight to the pedestal is the same as the position of the hand while holding the racket. The upper extremity rapidly rotates when the shuttlecock moves to the front of the player. Sequentially, it continues with the rotation of the hip, shoulder, and elbow joints (9). The same is performed with a forehand smash; the shuttlecock needs to be hit at the highest possible position. Furthermore, a flexible and strong wrist flexion motion is essential for producing a hard and targeted stroke (10). The application of motion mechanics principles is essential for producing a smash that provides maximum strength, speed, and accuracy to stop the opponent's movements and generate points (11).

Owing to the lack of backhand smashes, different studies tried to analyze almost the same motion patterns to add broader insights on tennis sports such as serve, smash, backhand, and forehand drive techniques. According to AbianVicen (12), a one-handed backhand drive is supported not only by the velocity of trunk rotation. It is determined by the amount of momentum and force movement generated from the shoulder and wrist joints. This drive involves the motion of body segments such as the legs, hips, trunk, upper arms, forearms, and hands (13). The velocity of maximal shoulder external rotation and the backswing of the upper arm are the main factors in generating a greater force when making a backhand drive (14).

Genevois (15) have reported that in the advanced player group, the maximum speed of the racket is obtained from the strength of the upper arm force. Meanwhile, in the novice group, the maximum speed is obtained from the motion of the wrist and elbow. During the one- handed backhand drive, the velocity of hip rotation significantly contributes to that of the other upper limb joints (16). Meanwhile, forehand smash requires harmonious coordination of body motions from the strength generated by the trunk, shoulders, arms, and wrists (17). To produce an effective smash, the biomechanics principles should be implemented in the phase of motion sequences. These include the preparation phase, backswing, forward swing, racket impact with the shuttlecock, and follow-through motion phase (18). Nesbit (19) indicated the importance of wrist flexion, forearm pronation, and upper arm rotation. In addition, the "kinetic chain movement" principle will produce an effective and efficient smash. The study by (20) reported that these joints and segments affected one another during the movement. When one is in motion, it creates a chain of events that affects the movement of neighboring joints and segments. Furthermore, the optimal performance in conducting a forehand smash depends on the motion of body segments that work in a harmonious motion chain sequence (12).

Based on the above mentioned background explanation, this study aims to analyze the movement of backhand and forehand smash techniques in badminton in three dimensions using the motion kinematics approach.

\section{MATERIALS AND METHODS}

Method and Design. The method used is a descriptive and quantitative approach. The descriptive method aims to systematically and accurately describe facts about certain parameters that are the center of attention.

Participants. The sample used in this study included 24 male badminton players with excellent skills who joined the university student activity unit; their average age was $19.4 \pm 1.6$ years, height of $1.73 \pm 0.12 \mathrm{~m}$, and weight of 62.8 $\pm 3.7 \mathrm{~kg}$. Furthermore, purposive sampling was used; all participants provided their written consent on a form that was previously given to them; in addition, the participants confirmed that they were not injured. Before the test, they received technical explanations related to the implementation of procedures in a comprehensive manner. The data collection test was conducted in the badminton field sports hall building, Faculty of Sports and Health Education, Indonesia University of Education. 


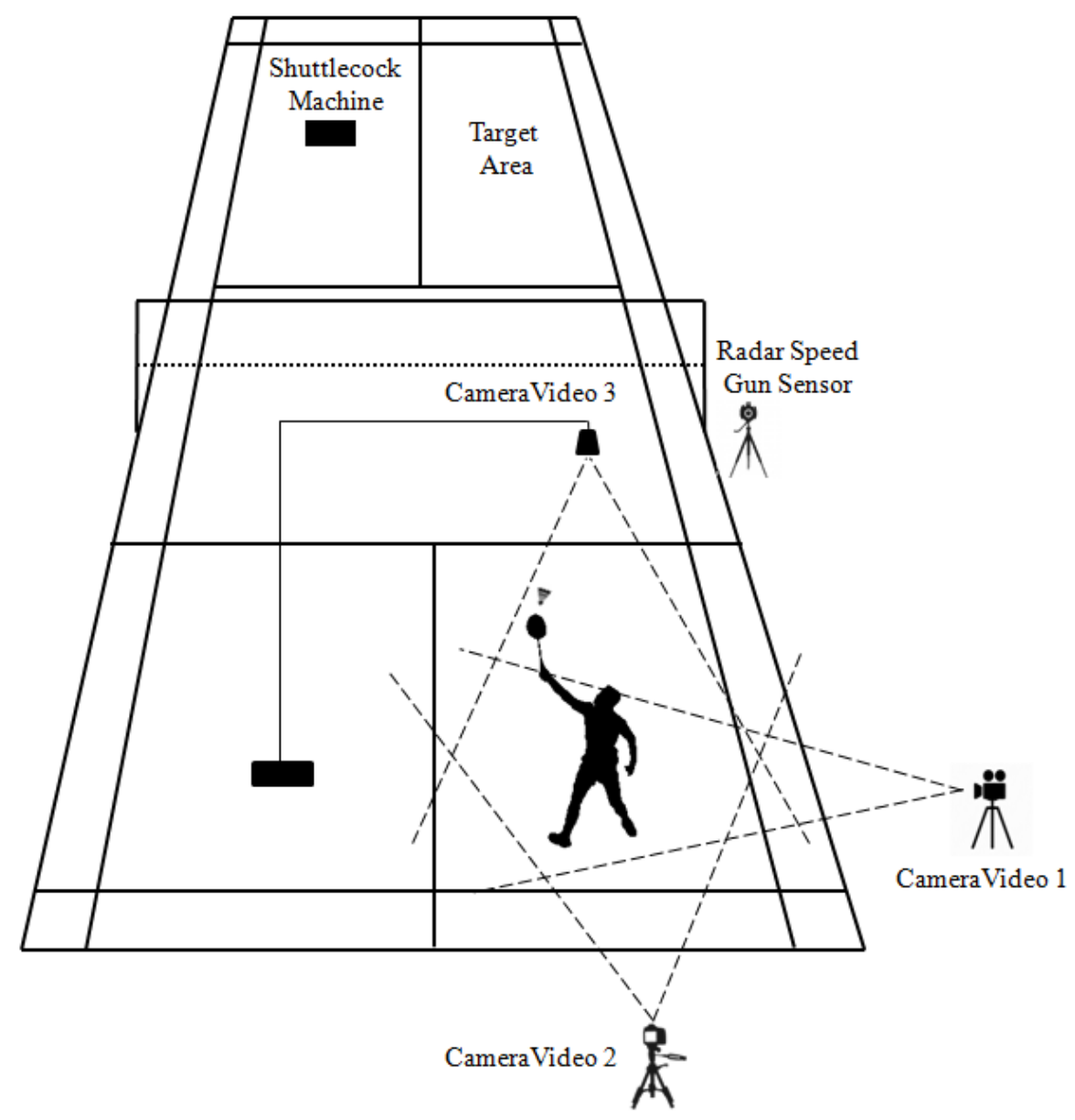

Figure 1. Schematic Diagram of the Setup Used to Collect the Data

Instruments. The instruments used were three video cameras (Panasonic Handycam HC-V100 Full HD, Japan), a three-dimensional calibration, a 3D motion analysis software (Frame DIAZ IV, Japan), one set of manual markers, a shuttlecock shooting machine (Plypower 143, Indonesia), and a radar speed gun (Bushnell Speed gun 101911, Italy).

Procedures. Before the test, the participants warmed up for approximately $15 \mathrm{~min}$. To be more comfortable and quickly adapt, the warm-up was followed by performing overhead backhand and forehand smashes using their racket. Subsequently, all participants were asked to execute 8 forehand and 8 backhand smash strokes to determine the mean velocity value in $\mathrm{km} / \mathrm{h}$.

Figure 1 shows the schematic diagram of field data collection. The ball speed was measured using a radar speed gun with a shutter speed of $250 \mathrm{~Hz}$. It was placed near the net at the distance of $45 \mathrm{~cm}$ outside the field line. In addition, video camera 1 was placed on the right side of the field at the distance of $2.5 \mathrm{~m}$ perpendicular to the position where the subject was standing. Video camera 2 was positioned behind the field line parallel to the subject area at the distance of $3 \mathrm{~m}$ from the player's position. Video camera 3 was placed above the position where the subject was standing in a perpendicular position parallel to the subject area. The three video cameras were set by the users according to the needs of the study characteristics. The camera settings used were as follows: frame rate of $250 \mathrm{~Hz}$, shuttle speed of $250 \mathrm{~s}$, and exposure time of $1 / 1200 \mathrm{~s}$. The calibration and data processing analyzed in three dimensions were conducted using the direct linear transformation structure method developed by Aziz Abdel (21).

Data Analysis. This study used the SPSS version 22.0 software (SPSS Inc., Chicago, IL), where the average and standard deviation were calculated as initial data for further calculations of normality, homogeneity, and hypothesis tests. To test the hypothesis, a one-way analysis of variance approach was used. This analysis allowed to calculate the level of difference 
between backhand and forehand overhead smashes with significant differences of 0.05 . The three-dimensional coordinate data of the signs affixed to each part of the player's joints were adjusted using the Butterworth low-pass filter method approach. This procedure was performed with a cut-off frequency of $15 \mathrm{~Hz}$ and used the residual analysis technique (22).

Kinematics Parameters. To obtain the kinematic parameters of an overhead smash motion, a model was developed based on the anatomical principles of the body (Figure 2).
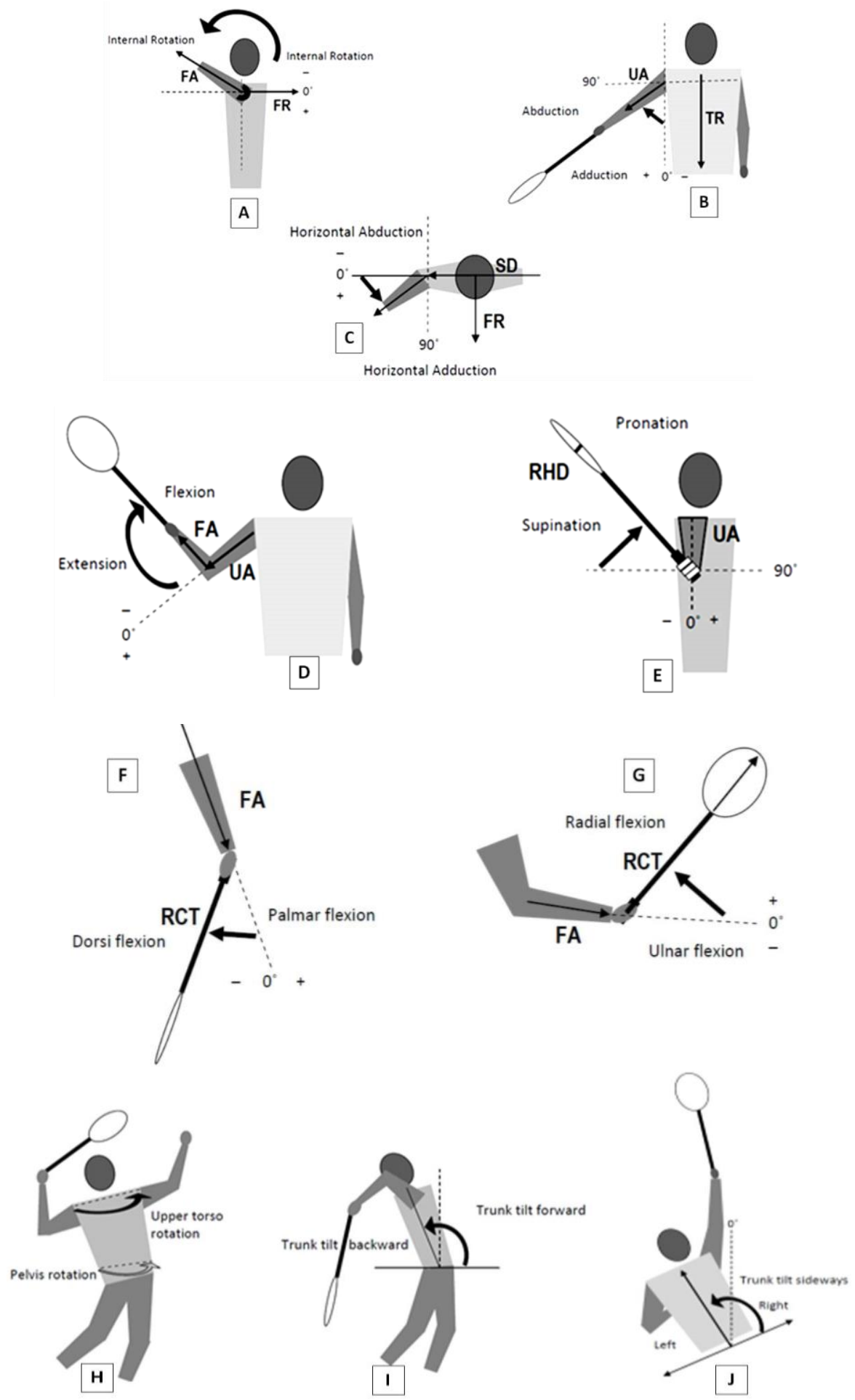

Figure 2. Kinematic Parameters of the Upper Limb Joints (Source: Rusdiana, 2020) 
Initially, the shoulder joint performs 3 movements, i.e., internal-external rotation (A), abduction-adduction (B), and horizontal abductionadduction (C). The elbow joint performs 2 movements, i.e., flexion-extension (D) and forearm pronation-supination (E). The wrist joint performs 2 movements, i.e., palmar-dorsiflexion (F) and radialulnar flexion $(\mathrm{G})$. The next movements are upper torso rotation and pelvis rotation $(\mathrm{H})$, trunk tilt forward and trunk tilt backward (I), as well as trunk tilt left and right sideways (J).

\section{RESULTS}

Table 1 shows the data on the difference in ball speed and changes in the kinematics of motion during backhand and forehand smashes.

Table 1. Kinematic Parameters of the Maximal Shoulder External Rotation

\begin{tabular}{lccc}
\hline \multirow{2}{*}{ Kinematic Parameter Analysis } & Backhand & Forehand & \multirow{2}{*}{ P-Value } \\
\cline { 2 - 3 } Shuttlecock velocity (km/h) & Means \pm SD & Means \pm SD & $0.035^{*}$ \\
Shoulder external rotation $(\mathbf{d e g})$ & $112 \pm 5.7$ & $158 \pm 3.5$ & $0.048^{*}$ \\
Shoulder abduction $(\mathbf{d e g})$ & $-122 \pm 3.5$ & $-169 \pm 4.2$ & 1.433 \\
Shoulder horizontal adduction (deg) & $101 \pm 1.2$ & $106 \pm 1.4$ & 1.248 \\
Elbow flexion (deg) & $7 \pm 0.83$ & $9 \pm 0.96$ & 0.983 \\
Radio-ulnar pronation (deg) & $94 \pm 1.1$ & $102 \pm 1.3$ & 1.778 \\
Wrist palmar flexion (deg) & $7 \pm 1.1$ & $12 \pm 1.3$ & $0.037 *$ \\
Trunk tilt backward (deg) & $-23 \pm 2.1$ & $24 \pm 3.1$ & 1.942 \\
Trunk tilt sideways left (deg) & $21 \pm 3.5$ & $21 \pm 1.6$ & 1.572 \\
\hline
\end{tabular}

*Significant differences at the $\mathbf{0 . 0 5}$ level

Table 2. Kinematic Analysis Parameters of the Maximum Angular Velocity

\begin{tabular}{lccc}
\hline \multirow{2}{*}{ Kinematic Parameter Analysis } & Backhand & Forehand & \multirow{2}{*}{ P-Value } \\
\cline { 2 - 3 } Shoulder internal rotation $(\mathbf{d e g} / \mathbf{s})$ & Means $\mathbf{\text { SD }}$ & Means \pm SD & $0.042^{*}$ \\
Upper torso rotation $(\mathbf{d e g} / \mathbf{s})$ & $1623 \pm 3.5$ & $2111 \pm 4.2$ & 1.252 \\
Pelvis rotation $(\mathbf{d e g} / \mathbf{s})$ & $761 \pm 1.2$ & $782 \pm 1.4$ & 1.566 \\
Elbow extension $(\mathbf{d e g} / \mathbf{s})$ & $421 \pm 0.8$ & $429 \pm 0.9$ & $0.035^{*}$ \\
Forearm Supination $(\mathbf{d e g} / \mathbf{s})$ & $523 \pm 1.1$ & $995 \pm 1.3$ & $0.024^{*}$ \\
Wrist dorsi flexion $(\mathbf{d e g} / \mathbf{s})$ & $642 \pm 1.1$ & $894 \pm 1.3$ & 0.983 \\
Trunk tilt forward $(\mathbf{d e g} / \mathbf{s})$ & $793 \pm 2.1$ & $195 \pm \pm 2.4$ & 1.482 \\
\hline
\end{tabular}

*Significant differences at the 0.05 level

Table 1 shows significant differences in three variables of the nine kinematic parameters analyzed in the maximal shoulder external rotation phase. These include shuttlecock velocity $(\mathrm{P}=0.035)$, shoulder external rotation $(\mathrm{P}=$ 0.048), and wrist palmar flexion $(\mathrm{P}=0.037)$. These results show that the three variables for the forehand smash have greater values than those for the backhand smash.

Table 2 shows significant differences in three variables of the seven kinematic parameters analyzed in the maximum angular velocity phase during the forehand smash. These include the speed of the shoulder internal rotation $(p=0.042)$, elbow extension $(p=0.035)$, and forearm supination $(p=0.024)$. These results show that the three variables for the forehand smash have greater values than those for the backhand smash.

\section{DISCUSSION}

The obtained results showed a significant difference in the maximum speed of the shuttlecock produced during the forehand smash compared to that during the backhand smash. Other studies showed a positive contribution between shuttlecock speed and wrist angular velocity when making backhand and forehand smashes. Meanwhile, the sequence pattern of upper limb joint rotation at the beginning of the backswing phase up to the moment of impact is similar in the two smash techniques. The shoulder joint rotation velocity was higher than that of the elbow joint. The wrist flexion angular velocity was smaller than the elbow angular velocity. These results are consistent with those of Creveaux (23), where the upper limb motion sequence starts with the rotation of the shoulder, elbow, and wrist joints during backhand drives in tennis. According to Rota (24), the major contribution to racket speed is obtained from the forearm supination rotation motion. (25) have stated that the combination of shoulder internal rotation and forearm supination provides approximately a 53\% support for the shuttlecock speed during an overhead forehand smash. This result is related to the backhand smash technique. 
This result shows that forearm supination and upper arm lateral rotation provide the maximum

bearing capacity to the speed of the racket swing before the impact occurs (26).

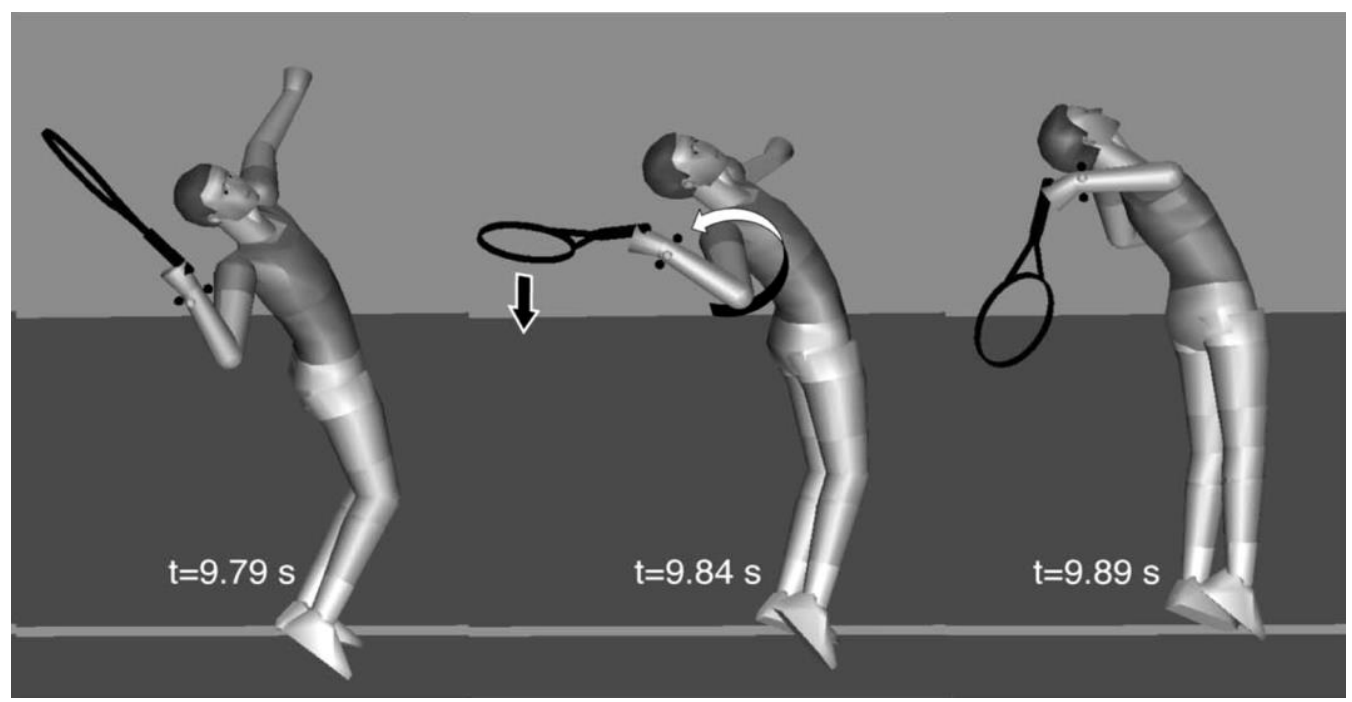

Figure 3. Contribution of Shoulder Maximal External Rotation When the Racket is Swinging Backward (Source: Gordon) (27)

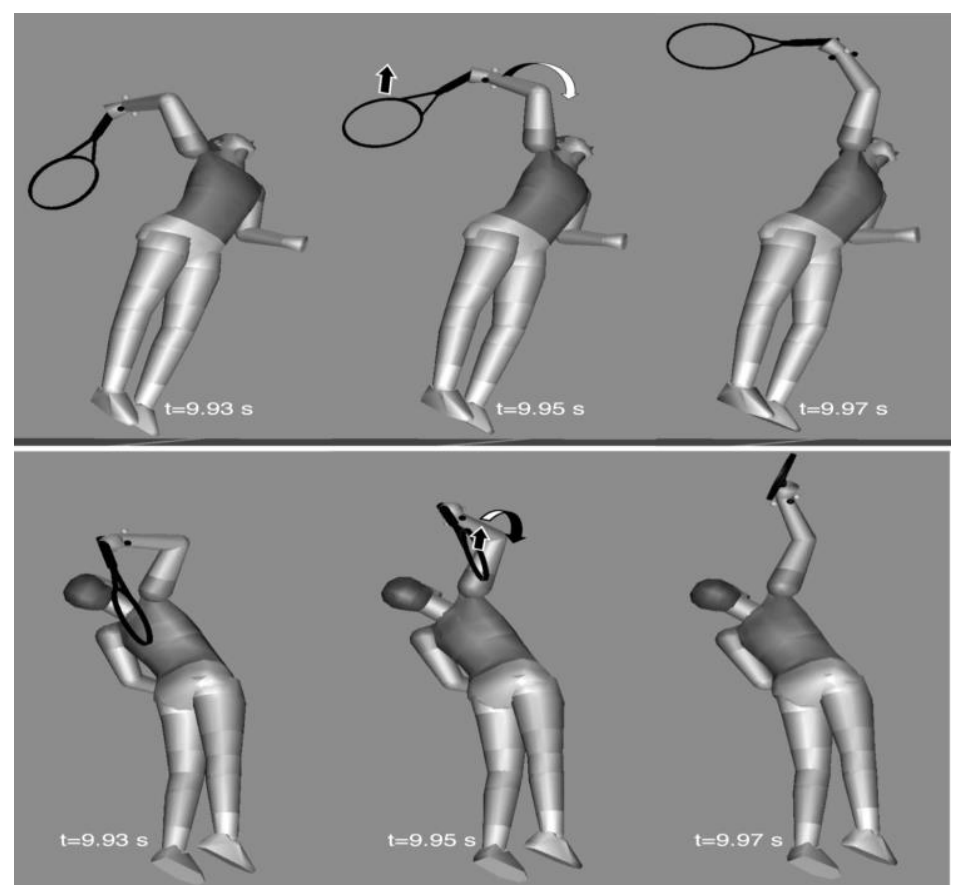

Figure 4. Elbow Flexion-Extension Movement (Source: Gordon) (27)

A series of motion patterns in overhead forehand and backhand smashes require linear and circular velocity as well as an acceleration of the body movement, shuttlecock, and racket swing. There are few studies on badminton that explain the movements of forehand and backhand overhead smash stroke techniques. However, the study by Gordon (27) analyzed the contribution of upper limb joint rotation velocity during the tennis serve. It has been stated that the backward maximal shoulder external rotation is the initial momentum, which produces a larger forward shoulder internal rotation force (28). This movement results in a greater racket speed, as shown in Figure 3.

Furthermore, the joint velocity during elbow extension is significantly higher, especially during the forehand smash. This result is consistent with the one in the study conducted by Reid (29) on the tennis serve. It has been reported that elbow joint provides positive support for racket speed. During the elbow extension motion, 
the faster the elbow rotates, the higher is the produced force on the motion of the upper arm and racket. This occurs before the impact on the shuttlecock, as shown in Figure 4. Furthermore, the elbow extension motion contributes approximately $30 \%$ to the racket speed (6). Another joint rotation that affects racket speed is the arm velocity during the radio-ulnar pronation motion (27). This movement pattern is especially present in the group of players with high technical skills. Meanwhile, novices usually do not perform this motion. Therefore, it is not surprising that professional players produce shuttlecock speeds that are much greater than those of amateurs.

\section{CONCLUSION}

From the obtained results, it is concluded that the shuttlecock speed during the forehand smash is greater than that during the backhand smash. During maximal shoulder external rotation, the forehand smash has a significant difference in three variables including shuttlecock velocity, shoulder external rotation, and wrist palmar flexion. Furthermore, shoulder internal rotation, elbow extension, and forearm supination at maximum angular velocity were higher when performing a forehand smash. The shoulder internal rotation and elbow joint velocity as well as forearm supination significantly contribute to the shuttlecock speed when performing the twostroke techniques.

\section{APPLICABLE REMARKS}

- The smash is a shot hit with power and speed down to your opponent's court. The average number of smashes executed in one match in the men's single category was 69 strokes, while for the women's singles category it was 51 strokes in All England Championship 2015.

- The technique to perform the badminton backhand and forehand smashes is very different from tennis or squash. In badminton, the backhand and forehand stroke can be used to perform powerful shots such as a tennis serve to get points.

- Before hitting the backhand smash, make sure that your arm is close to your body so as to get a better swing while hitting the shuttlecock. Use your non-racket arm to help you balance.

- The follow-through phase is an important movement. Complete the swing action all the way through. Use your non-racket arm to maintain balance as you may lose balance while performing this stroke.

\section{ACKNOWLEDGMENTS}

The authors are grateful to the Universitas Pendidikan Indonesia, the Ministry of Research, Technology, and Higher Education, INDONESIA for their supports.

\section{CONFLICTS OF INTEREST}

The authors declare no conflict of interest regarding the publication of this study.

\section{REFRENCES}

1. Kuntze G, Mansfield N, Sellers W. A biomechanical analysis of common lunge tasks in badminton. $J$ Sports Sci. 2010;28(2):183-191. doi: 10.1080/02640410903428533 pmid: 20391092

2. Chow JY, Seifert L, Herault R, Chia SJ, Lee MC. A dynamical system perspective to understanding badminton singles game play. Hum Mov Sci. 2014;33:70-84. doi: 10.1016/j.humov.2013.07.016 pmid: 24075690

3. Barreira J, Chiminazzo JGC, Fernandes PT. Analysis of point difference established by winners and losers in games of badminton. Int $J$ Performance Anal Sport. 2016;16(2):687-694. doi: 10.1080/24748668.2016.11868916

4. Lam WK, Wong DW, Lee WC. Biomechanics of lower limb in badminton lunge: a systematic scoping review. PeerJ. 2020;8:e10300. doi: 10.7717/peerj.10300 pmid: 33194445

5. McErlain-Naylor SA, Towler H, Afzal IA, Felton PJ, Hiley MJ, King MA. Effect of racket-shuttlecock impact location on shot outcome for badminton smashes by elite players. J Sports Sci. 2020;38(21):24712478. doi: 10.1080/02640414.2020.1792132 pmid: 32662349

6. Martin C, Sorel A, Touzard P, Bideau B, Gaborit R, DeGroot H, et al. Can the Open Stance Forehand Increase the Risk of Hip Injuries in Tennis Players? Orthop $J$ Sports Med. 2020;8(12):2325967120966297. doi: 10.1177/2325967120966297 pmid: 33354579

7. Rusdiana A, Subarjah H, Imanudin I, Kusdinar Y, M Syahid A, Kurniawan T. Effect of Fatigue on Biomechanical Variable Changes in Overhead Badminton Jump Smash. Annal Appl Sport Sci. 2020;8(3):0-0. doi: 10.29252/aassjournal.895 
8. Sakurai S, Ohtsuki T. Muscle activity and accuracy of performance of the smash stroke in badminton with reference to skill and practice. J Sports Sci. 2000;18(11):901-914. doi: 10.1080/026404100750017832 pmid: 11144867

9. Li S, Zhang Z, Wan B, Wilde B, Shan G. The relevance of body positioning and its training effect on badminton smash. J Sports Sci. 2017;35(4):310-316. doi: 10.1080/02640414.2016.1164332 pmid: 27043765

10. Miller R, Felton PJ, Mcerlain-Naylor SA, Towler H, King MA. Optimum Performance in the Badminton Jump Smash. 19702013.

11. Ooi CH, Tan A, Ahmad A, Kwong KW, Sompong R, Ghazali KA, et al. Physiological characteristics of elite and sub-elite badminton players. $J$ Sports Sci. 2009;27(14):1591-1599. doi: 10.1080/02640410903352907 pmid: 19967588

12. Abian-Vicen J, Castanedo A, Abian P, Sampedro J. Temporal and notational comparison of badminton matches between mens singles and womens singles. Int J Performance Anal Sport. 2013;13(2):310-320. doi: 10.1080/24748668.2013.11868650

13. Alexandros M, Christina K, Nikolaos G, Konstantinos M. Effectiveness of backhand with one and two hands in teaching adult men beginners in tennis. J Physic Educat Sport. 2013;13(3):415-418. doi: 10.7752/jpes.2013.03066

14. Kolman NS, Kramer T, Elferink-Gemser MT, Huijgen BCH, Visscher C. Technical and tactical skills related to performance levels in tennis: A systematic review. J Sports Sci. 2019;37(1):108-121. doi: 10.1080/02640414.2018.1483699 pmid: 29889615

15.Genevois C, Reid M, Rogowski I, Crespo M. Performance factors related to the different tennis backhand groundstrokes: A review. J Sport Sci Med. 2014;14(1):194-202.

16. Wu SK, Gross MT, Prentice WE, Yu B. Comparison of ball-and-racquet impact force between two tennis backhand stroke techniqes. J Orthop Sports Phys Ther. 2001;31(5):247-254. doi: 10.2519/jospt.2001.31.5.247 pmid: 11352191

17. Mavvidis A, Metaxas T, Riganas C, Koronas K. Speed differences between forehand and backhand in intermediate tennis players. Kinesiol. 2005;37:159-163.

18.Phomsoupha M, Laffaye G. Shuttlecock velocity during a smash stroke in badminton evolves linearly with skill level. Comput Methods Biomech Biomed Engin. 2014;17 Suppl 1:140-141. doi: 10.1080/10255842.2014.931550 pmid: 25074204

19.Nesbit SM, Elzinga M, Herchenroder C, Serrano M. The effects of racket inertia tensor on elbow loadings and racket behavior for central and eccentric impacts. J Sport Sci Med. 2006;5(2):304-317.

20.Taha Z, Hassan MSS, Yap HJ, Yeo WK. Preliminary Investigation of an Innovative Digital Motion Analysis Device for Badminton Athlete Performance Evaluation. Procedia Engin. 2016;147(461-5). doi: 10.1016/j.proeng.2016.06.341

21.Hong Y, Wang SJ, Lam WK, Cheung JT. Kinetics of badminton lunges in four directions. J Appl Biomech. 2014;30(1):113-118. doi: 10.1123/jab.2012-0151 pmid: 23878207

22.Iino Y, Kojima T. Kinetics of the upper limb during table tennis topspin forehands in advanced and intermediate players. Sports Biomech. 2011;10(4):361-377. doi: 10.1080/14763141.2011.629304 pmid: 22303787

23.Creveaux T, Dumas R, Hautier C, Rogowski I. Joint kinetics to assess the influence of the racket on a tennis player's shoulder. Journal of Sports Science and Med. 2013;12(2):259-266.

24.Rota S, Morel B, Saboul D, Rogowski I, Hautier C. Influence of fatigue on upper limb muscle activity and performance in tennis. $J$ Electromyogr Kinesiol. 2014;24(1):90-97. doi: 10.1016/j.jelekin.2013.10.007 pmid: 24239164

25.Rogowski I, Creveaux T, Cheze L, Mace P, Dumas R. Effects of the racket polar moment of inertia on dominant upper limb joint moments during tennis serve. PLoS One. 2014;9(8):e104785. doi: 10.1371/journal.pone.0104785 pmid: 25117871

26.Fu L, Ren F, Baker JS. Comparison of Joint Loading in Badminton Lunging between Professional and Amateur Badminton Players. Appl Bionics Biomech. 2017;2017:5397656. doi: 10.1155/2017/5397656 pmid: 28694684

27. Gordon BJ, Dapena J. Contributions of joint rotations to racquet speed in the tennis serve. J Sports Sci. 2006;24(1):31-49. doi: 10.1080/02640410400022045 pmid: 16368612 
28. Maeda RS, Cluff T, Gribble PL, Pruszynski JA. Compensating for intersegmental dynamics across the shoulder, elbow, and wrist joints during feedforward and feedback control. J Neurophysiol. 2017;118(4):1984-1997. doi: 10.1152/jn.00178.2017 pmid: 28701534

29.Reid M, Elliott B, Crespo M. Mechanics and learning practices Associated with the Tennis forehand: A review. J Sport Sci Med. 2013;12(2):225-231. 